\title{
L'observation spatiale dans le programme AMIMA
}

\section{Résumé}

L'observation spatiale est largement utilisée dans le cadre du programme AMMA pour des objectifs variés : études de processus, de variabilité climatique, applications sociétales. Ces travaux concernent l'atmosphère, l'océan, les surfaces continentales et les interactions entre ces milieux. Cependant, le cadre expérimental AMMA s'est également montré favorable pour améliorer les méthodes d'exploitation des données spatiales : validation de mesures de satellites récemment lancés ou pour lesquels il n'y avait pas eu de validation dans cette région, élaboration de nouvelles méthodes d'utilisation, pour obtenir des variables du milieu avec précision (précipitations, par exemple), ou pour permettre l'exploitation de longues séries de données.

\section{Abstract}

\section{Use of space observation in the AMMA programme}

Spaceborne Earth observation has been widely used within the AMMA program, for various studies: process, climate variability, society impact applications. Studies are being performed in the atmosphere, ocean, land surface domains as well as interactions between them. However, the AMMA experimental framework has offered the opportunity to develop space data methodologies: data validation of new sensors, specific regional validation benefiting from an exceptional data set, unusual in this region, development of new methodologies to get either variables more accurately (e.g. precipitations) or to exploit long-term series.

\author{
Laurence Eymard (1), Christian Baron $^{(2)}$, Guy Caniaux ${ }^{(3)}$, \\ Cyrille Flamant $^{(4)}$, Laurent Kergoat ${ }^{(5)}$, Fatima Karbou ${ }^{(6)}$, \\ Jacques André Ndione ${ }^{(7)}$, Thierry Pellarin ${ }^{(8)}$, Nadège Martinyy ${ }^{(9)}$, \\ Johanna Ramarohetra ${ }^{(1)}$, Elodie Vintrou ${ }^{(2)}$ et Rémy Roca $^{(10)}$ \\ ( 1) LOCEAN/IPSL, UPMC/CNRS/IRD/MNHN, Paris, France \\ ( 2) CIRAD UMR TETIS, Montpellier, France \\ ( 3) CNRM/GAME, Météo-France/CNRS, Toulouse, France \\ (4) LATMOS/IPSL, UVSQ/UPMC/CNRS, Paris, France \\ ( 5) GET, UPS/CNRS/IRD, Toulouse, France \\ (6) CNRM/CEN, Météo-France, Grenoble, France \\ (7) CSE, Dakar, Sénégal \\ ( 8) LTHE, UJF/CNRS/IRD/INPG, Grenoble, France \\ ( 9) CRC, Université de Bourgogne/CNRS, Dijon, France \\ (10) LMD/IPSL, ENS/X/UPMC/CNRS, Paris, France
}

P our comprendre les mécanismes de la variabilité climatique associés à la mousson africaine, étudier les échanges surface-atmosphère, analyser les processus du bilan hydrique régional, le programme AMMA (Analyses multidisciplinaires de la mousson africaine) a mis en place un dispositif d'observations sur le terrain sans précédent et animé une forte activité de modélisation numérique. Cependant, l'immensité du territoire concerné (l'Afrique de l'Ouest jusqu'au Sahara, les bassins océaniques entourant le continent africain, voire la totalité de la ceinture tropicale et même le globe pour l'étude du climat) et la variété des échelles de temps (de celle de la turbulence de couche limite jusqu'à l'échelle décennale et au-delà) a imposé une stratégie d'observation incluant l'observation spatiale dès les premières réflexions qui ont donné naissance à AMMA.

Les connaissances scientifiques qui permettent cette utilisation des mesures satellitaires sont nombreuses : on peut citer par exemple le suivi des systèmes convectifs africains avec les données dans l'infrarouge de Météosat depuis le début des années 1980 (Arnaud et al., 1992 ; Fiolleau et al., 2009), l'exploitation des données dans le visible pour le suivi de la végétation (par exemple Lo Seen et al., 1993 ; Anyamba et al., 2005 ; Frison et Mougin, 1996), l'observation des émissions de $\mathrm{CO}$ et autres gaz liés aux feux de brousse (Mari et al., 2008) ou encore l'étude de la variabilité océanique en surface à l'aide de l'altimétrie ou de la température de surface (par exemple Mayer et al., 2001).

La base de données AMMA a été construite pour favoriser l'utilisation des données spatiales, avec comme objectif que ces données ne soient pas seulement utilisées par les spécialistes de chaque type de mesure, mais plus largement pour toutes les applications scientifiques : observation régionale en complément de l'observation locale, validation de simulations, analyses de variabilité et nouvelles applications comme l'occurrence de maladies, la gestion des sols... (voir l'article de Fleury et al., dans ce numéro, p. 90-93)

À l'inverse, le programme AMMA a offert des opportunités pour mieux interpréter les mesures spatiales dans cette région du globe : d'une part, le développement de nouvelles méthodes d'exploitation des satellites existants a pu être entrepris en bénéficiant de 
mesures in situ pour la validation, d'autre part, des mesures ont été effectuées après le lancement de nouvelles missions spatiales pour permettre une validation scientifique dans un contexte climatique bien étudié.

Cet article décrit les principales études et résultats fondés sur l'utilisation des données spatiales. Nous montrerons d'abord comment AMMA a contribué à la validation ou la préparation de plusieurs missions spatiales internationales. Puis, nous verrons quelles sont les questions qui ont nécessité des développements spécifiques et les nouvelles idées que AMMA a suscitées. L'exploitation scientifique des données spatiales sera ensuite illustrée par quelques exemples dans des domaines importants du programme. Dans une dernière partie, l'apport de l'observation spatiale au développement de nouvelles thématiques reliant environnement et société sera présenté. Certaines études ont nécessité à la fois de la validation instrumentale et du développement méthodologique avant toute exploitation scientifique. Cet aspect sera illustré.

L'encadré ci-contre résume les principales missions spatiales et les instruments mentionnés dans cet article.

\section{La validation des données de missions spatiales grâce aux données de terrain AMIMA}

L'implantation du dispositif de mesure en différents points du continent (Redelsperger et al., 2007) le long d'un gradient nord-sud de végétation et dans des zones climatiques différentes a offert la possibilité d'adjoindre un volet de validation directe ou d'études reliées à certaines missions spatiales, avec le soutien du Centre national d'études spatiales (CNES). Les missions concernées sont principalement CALIPSO (CloudAerosol Lidar and Infrared Pathfinder Satellite Observation; Pelon et al., 2011), lancée en 2006, et SMOS (Soil Moisture and Ocean Salinity ; Kerr et al., 2010), lancée en 2009.

Rappelons que les mesures des satellites ne fournissent pas directement les variables mesurables comme les capteurs in situ : les instruments spatiaux mesurent un rayonnement, soit émis naturellement par le milieu, soit

\section{Missions spatiales et instruments cités}

A-Train : ensemble de 5 satellites (Aqua, Aura, PARASOL, CALIPSO et CLOUDSAT) se suivant sur l'orbite du satellite météorologique Aqua.

Aqua, lancé en 2002, emporte :

- I'instrument MODIS : imageur visible infrarouge $(0,4$ à 14 um), de résolution 250 m ; - les sondeurs AMSU-A et -B : instruments profileurs de température et humidité en micro-onde, fréquences 23 à $183 \mathrm{GHz}$ (sur Aqua et les autres satellites météorologiques américains NOAA-n) ;

- I'instrument AMSR-E : imageur micro-onde pour la surface et l'atmosphère, fréquences de 6 à $89 \mathrm{GHz}$;

Aura : lancé en 2004, emporte quatre instruments pour l'étude de la chimie atmosphérique :

- HIRDLS (High Resolution Dynamics Limb Sounder) mesurant le rayonnement infrarouge émis par l'ozone, la vapeur d'eau, le méthane;

MLS (Microwave Limb Sounder) mesurant la température et la vapeur d'eau dans la haute atmosphère ;

OMI (Ozone Monitoring Instrument) qui a succédé à TOMS et qui utilise le rayonnement ultraviolet et la lumière visible pour produire quotidiennement des cartes à haute résolution d'ozone et d'aérosols ;

- TES (Tropospheric Emission Spectrometer) qui mesure l'ozone présent dans la troposphère à l'aide des émissions infrarouges ainsi que le monoxyde de carbone, le méthane et les oxydes d'azote.

En plus de l'ozone, ses instruments fournissent la concentrations en divers gaz ( $\mathrm{NO}_{2}$ $\mathrm{SO}_{2}, \mathrm{BrO}, \mathrm{OClO}$ ) et des informations sur les aérosols.

PARASOL : lancé en 2004, emporte le radiomètre POLDER qui mesure les caractéristiques directionnelles et la polarisation de la lumière réfléchie par les surfaces terrestres et l'atmosphère.

CALIPSO : lancé en 2006, comprend I'instrument CALIOP (Cloud-Aerosol Lidar with Orthogonal Polarization) - lidar aérosols et nuages à visée verticale ainsi qu'une caméra fonctionnant en lumière visible à ouverture grand champ et un radiomètre imageur infrarouge à 3 canaux.

CLOUDSAT : lancé en 2006, comprend un radar profileur dans les nuages, fréquence $35 \mathrm{GHz}$

SMOS (Soil Moisture and Ocean Salinity) : satellite transportant un radiomètre microonde expérimental de fréquence 1,4 GHz, pour la détermination de l'humidité du sol et de la salinité de surface de l'océan (lancé en 2009).

Météosat (jusqu'en 2002) et MSG (Météosat seconde génération) depuis : satellites météorologiques géostationnaires opérationnels, centrés au-dessus du méridien $0^{\circ}$, emportant SEVIRI, un système imageur visible et infrarouge.

Landsat : série de satellites d'imagerie de la surface en visible (résolution de $30 \mathrm{~m}$ ) et infrarouge (résolution 60 m), de 1972 à maintenant.

QuickSCAT : satellite pour la mesure du vent de surface sur les océans lancé en 1999, il emporte le radar diffusiométrique SeaWinds.

ERS1 et ERS2 : famille de satellites d'observation de la terre de l'Agence spatiale européenne (1991-2005) emportant plusieurs instruments pour l'océan, I'atmosphère et les surfaces continentales, notamment le diffusiomètre SCATT.

METOP : satellite météorologique européen défilant, lancé en 2005, emportant plusieurs instruments :

- les sondeurs micro-onde AMSU-A et MHS (similaire à AMSU-B) pour les profils de température et d'humidité

- le sondeur infrarouge IASI pour le profil de température, humidité et plusieurs constituants minoritaires ;

- le diffusiomètre ASCAT pour la mesure du vent de surface sur les océans.

TOMS (Total Ozone Mapping Spectrometer) : instrument de mesure de l'ozone atmosphérique (contenu total), fonctionnant dans l'ultraviolet. Il a été embarqué de 1978 à 2005 sur plusieurs missions (Nimbus-7, Meteor-3, Earth Probe).

TRMM (Tropical Rainfall Meausuring Mission) : satellite lancé en 1997, dédié aux précipitations, emportant entre autres le radar PR (Precipitation Radar, $35 \mathrm{GHz}$ ) et le radiomètre micro-onde multispectral à balayage conique TMI.

réfléchi ou diffusé à partir d'un signal émis depuis le satellite (radar ou lidar). La validation des résultats déduits des satellites est donc une activité essentielle après le lancement et tout au long de la vie de la mission spatiale, pour contrôler leur qualité. L'exemple des données d'ozone du capteur OMI 
(Ozone Monitoring Instrument) sur le satellite Aura est donné comme illustration de ce dernier aspect.

\section{SMOS: humidité des sols}

L'objectif de la mission SMOS est de fournir des cartes d'humidité des sols afin de mieux contraindre les modèles de circulation générale et décrire les conditions aux limites pour les modèles d'hydrologie à grande échelle. Les stations de mesure de l'humidité du sol installées en 2006 dans le cadre du programme AMMA ont été maintenues grâce au soutien des organismes, et notamment du CNES, afin de per- mettre une validation des mesures du satellite SMOS (de Rosnay et al., 2009). Depuis son lancement fin 2009, ses mesures sont analysées en confrontant les estimations satellitaires d'humidité du sol aux observations réalisées sur le terrain. Une cartographie mensuelle de l'humidité du sol sur l'ensemble de l'Afrique de l'Ouest (figure 1) a été réalisée par Gruhier et al. (2012).

\section{Calipso : nuages et aérosols}

La station TReSS (Transportable Remote Sensing Station), composée d'un lidar rétrodiffusion, d'un photomètre solaire et d'un ensemble de radiomètre et de capteurs in situ, a été déployée à Tamanrasset, en Algérie, de février à novembre 2006, dans le cadre de la campagne AMMA (Cuesta et al., 2008). Ce déploiement expérimental lourd a permis de contribuer à l'effort de validation des premiers produits opérationnels du lidar spatial CALIOP (Cloud-Aerosol Lidar with Orthogonal Polarization) embarqué sur la plateforme CALIPSO. Un des premiers résultats obtenus à partir des observations lidar de TReSS a été la mise en évidence, dès juin 2006, d'un décalage vertical de $500 \mathrm{~m}$ sur les profils de rétrodiffusion issus de CALIOP.

Figure 1 - Cartographie mensuelle de l'humidité du sol estimée à partir des mesures du satellite SMOS. On observe clairement l'évolution de la saison des pluies ainsi que l'extension spatiale du delta intérieur du Niger.

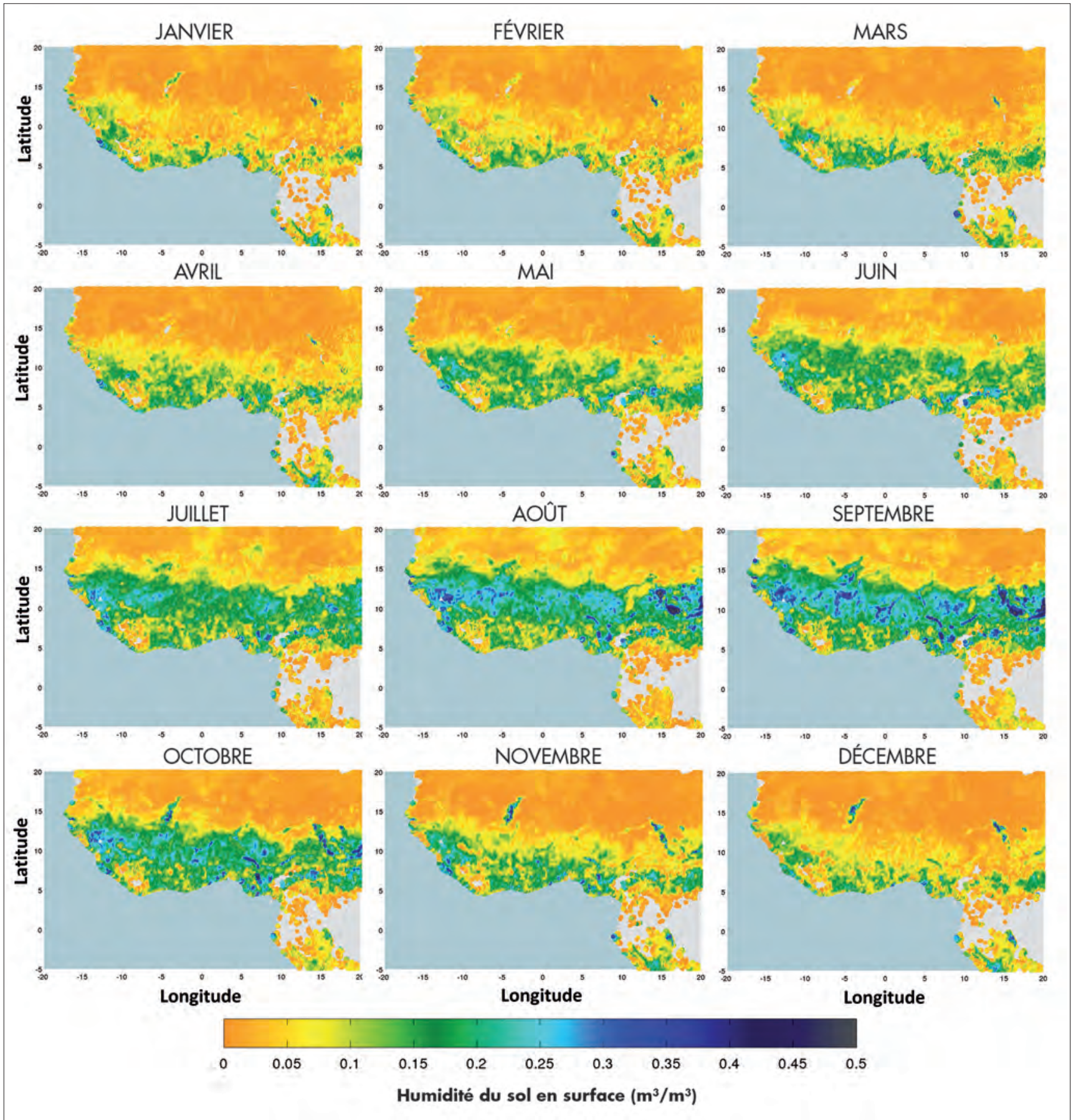


Cuesta et Flamant (2010) ont développé une méthode originale permettant la validation signal-à-signal des mesures lidar en visées opposées (le lidar spatial CALIOP vise au nadir et le lidar au sol au zénith), en s'affranchissant de l'ambiguïté associée au traitement lidar pour comparer les profils de rétrodiffusion et/ou d'extinction. Cette technique à deux flux peut être également utilisée pour la restitution des profils d'extinction et de rétrodiffusion pour les aérosols et les nuages. La méthode a été appliquée pour valider les observations CALIPSO de la couche limite saharienne, en utilisant les mesures sol de TReSS à Tamanrasset.

Par ailleurs, l'instrument aéroporté RALI (Protat et al., 2004) composé du radar RASTA $(95 \mathrm{GHz})$ et du lidar rétrodiffusion LNG a été embarqué à bord du Falcon 20 de SAFIRE au cours de la troisième période intensive d'AMMA, en fin d'été 2006, pour valider les instruments CALIOP et de CloudSat (Protat et al., 2009 et 2010). Quatre vols sous la trace des satellites ont été réalisés $(9,20,21$ et 22 septembre 2006). La première estimation de la diffusion multiple à l'intérieur du faisceau radar de Cloudsat a été effectuée, en comparant les mesures de RASTA très peu sensible à la diffusion multiple (faisceau étroit) à la mesure de CloudSat (Bouniol et al., 2008).

\section{Validation des données aérosols issues du capteur OMI}

Les données spatiales de contenu en aérosols (capteur OMI, Ozone Monitoring Instrument) sont des produits « opérationnels ». Leur utilisation pour des questions scientifiques particulières suppose au préalable de les valider dans le contexte considéré. Ainsi, dans le cadre de l'étude du lien entre aérosols désertiques et épidémies de méningites au Sahel (voir la dernière section), les indices d'absorption des aérosols (notés AI) issus du capteur OMI ont été validés au Niger, Mali et Burkina Faso (qui figurent parmi les pays les plus touchés par les épidémies de méningite) de 2006 à 2009 (en plein cœur du programme AMMA). Cette validation spécifique n'avait jamais été menée auparavant en Afrique de l'Ouest. Les mesures de terrain utilisées comme référence pour la validation des AI sont les épaisseurs optiques des aérosols (AOT) issues du réseau AERONET (Holben et al., 2001) d'une part, et les concentrations en surface des aérosols dont le diamètre est inférieur à $10 \mu \mathrm{m}$ $\left(\mathrm{PM}_{10}\right)$ issues des 3 stations de mesures du « transect de poussière sahélienne » (Marticorena et al., 2010) d'autre part. L'analyse de tous les jours communs de mesures d'AI et d'AOT sur la période 2006-2009 a permis de montrer le bon accord entre le produit satellitaire et la mesure sol (coefficient de corrélation variant entre 0,62 et de 0,68 selon les sites). Au pas de temps hebdomadaire, ce coefficient de corrélation est amélioré, variant de 0,70 à 0,78 selon les sites. L'AI à une résolution de $0,25^{\circ}$ semble donc être un bon indicateur du contenu en aérosols intégré sur la colonne atmosphérique. Deroubaix et al. (2012) montrent également que l'AI reproduit de manière satisfaisante le cycle saisonnier des aérosols (figure 2).

Le début de la saison des pluies est bien détecté (fin juin/début juillet), les anomalies deviennent négatives. La saison sèche est partagée en 3 périodes distinctes :

- celle pendant laquelle il n'y a pas ou peu d'aérosols désertiques dans l'atmosphère, d'août à novembre les anomalies sont majoritairement négatives ; - aérosols dans les basses couches de l'atmosphère de décembre à mars (Léon et al., 2009) : les produits représentatifs du contenu intégré des aérosols sur la colonne atmosphérique (AI ou AOT)

Figure 2 - Validation du cycle annuel moyen standardisé de l'Al-OMI (trait plein bleu) via les mesures d'AOT (trait pointillé bleu) et les mesures des concentrations en $\mathrm{PM}_{10}$ (trait plein rouge) sur la période 2006-2009, à Banizoumbou (Niger).

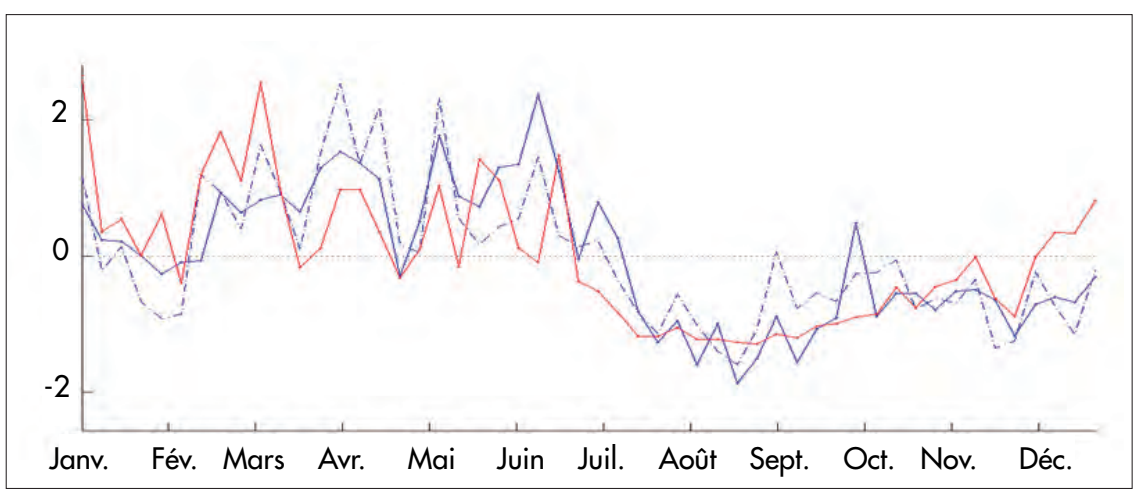

ont des valeurs systématiquement inférieures à celles des $\mathrm{PM}_{10}$;

- aérosols dans les hautes couches d'avril à juin : AI ou AOT sont quasi systématiquement supérieures à celles des $\mathrm{PM}_{10}$. En outre, les événements d'aérosols importants comme celui de mars 2006 durant la SOP AMMA (Slingo et al., 2006) peuvent être clairement détectés par l'AI, même si cette détection est modérée comparativement aux AOT et aux $\mathrm{PM}_{10}$.

\section{Le développement de nouvelles méthodes et approches dans le cadre d'AMIMA}

Dans la plupart des cas, les mesures sont traitées par les agences spatiales et mises à disposition des utilisateurs. Certaines équipes, directement impliquées dans une mission spatiale, contribuent à ces développements. Cependant, même pour des missions considérées comme « opérationnelles », il peut s'avérer nécessaire de développer de nouvelles méthodes d'exploitation des données. Dans le cadre d'AMMA, des questions centrales comme l'estimation des précipitations ou le suivi des caractéristiques de surface ont suscité de nouvelles études dédiées. D'autres travaux ont été menés pour permettre des études sur la variabilité interannuelle de la mousson avec différents instruments spatiaux.

\section{Précipitations}

L'obtention des précipitations par satellite a fait l'objet d'une évaluation approfondie grâce à l'ensemble des observations sol recueillies. Ces évaluations couvrent une large gamme d'échelles : moyenne mensuelle ou décadaire (Ali et al., 2005 ; Jobard et al., 2011), journalière et diurne (Roca et al., 2010a), instantanée (Kirstetter et al., 2012 ; Kacimi et al., 2012). Ces travaux concernent une large variété d'applications : météorologie (Roca et al., 2010a), hydrologie (Meynadier et al., 2010 ; Gosset et al., 2012) et étude de végétation et poussière (Pierre et al., 2011). Il ressort de ces analyses que les progrès réalisés au cours de la dernière décennie (voir Roca et al., 2010b, pour une perspective historique), quant à la qualité et l'exploitabilité de ces produits « pluie », permettent d'envisager dorénavant leur utilisation quantitative. 
De nouveaux développements algorithmiques ont vu le jour avec l'algorithme EPSAT (Bergès et al., 2010) et plus récemment l'algorithme TAPEER (Chambon, 2011) élaborés dans le contexte de préparation de la mission Megha-Tropiques. Ces algorithmes combinent l'information radiométrique des instruments micro-onde (TRMM en particulier) aux données IR des géostationnaires. TAPEER adjoint de plus à l'estimation du cumul de pluie journalier sur des régions de $1^{\circ}$ de côté une estimation de l'erreur associée (Chambon et al., 2012). Ces développements se sont appuyés sur des comparaisons quantitatives avec les observations au sol au Sénégal (réseau mis en place dans le cadre AMMA) et deux réseaux pluviographiques denses (sites AMMACATCH, voir Lebel et al., 2009) au Bénin et au Niger. Ces mêmes réseaux d'observation contribueront à la validation de la mission Megha-Tropiques, lancée en octobre 2010. La région de Niamey a d'ailleurs accueilli en 2010 une campagne de pré-validation algorithmique pour cette mission, combinant avion instrumenté et radar, afin de documenter la microphysique des gros systèmes convectifs et aider à l'ajustement de l'algorithme d'estimation des pluies BRAIN à partir des données microondes (Viltard et al., 2006).

La haute répétitivité de la mesure du satellite géostationnaire Météosat a permis de documenter les propriétés morphologiques des systèmes convectifs de mésoéchelle (taille du nuage, durée, heure de naissance, etc.) sur une période de plus de 25 ans (Fiolleau et al., 2009). Une série d'outils novateurs a fait émerger des définitions des classes des systèmes adaptées à l'étude de la mousson africaine (Fiolleau et al., 2009). Ce jeu de données unique est disponible sur la base de données AMMA et devrait permettre d'explorer les variations de l'occurrence des systèmes convectifs organisés sur plus de 30 ans.

Ces deux approches se sont alliées pour permettre une validation des simulations du modèle climatique LMDz en Afrique de l'Ouest tant aux échelles synoptiques (Ly, 2009) qu'à l'échelle du cycle diurne (Sane, 2010). Des cycles diurnes composites de précipitation ont été construits sur Dakar à l'aide du réseau sol en filtrant les événements convectifs propagatifs tirés de Météosat, offrant ainsi au modèle une observation qui correspond aux processus physiques à évaluer. Les comparaisons soulignent que le nouveau schéma physique de LMDz permet une reproduction fidèle du cycle diurne tant au niveau de l'heure de déclenchement que de la durée de la convection profonde. Les produits pluie satellitaires ont finalement permis d'effectuer le passage de l'échelle locale (Dakar) pour évaluer le processus à l'échelle régionale sur toute 1'Afrique de 1'Ouest (Sane et al., 2012).

\section{Suivi des aérosols}

La création de longues séries de données temporelles est indispensable pour mener à bien des études en climatologie. Actuellement, les AI issus des capteurs TOMS $\left(1 \times 1,25^{\circ}\right)$ et OMI $\left(0,25^{\circ}\right)$ n'offrent pas une série temporelle continue. En effet, la fin de la période TOMS (2002-2005) est entachée d'une dérive instrumentale qui conduit à des AI erronés (Bhartia et al., 2004). La création d'une série continue de données TOMS/OMI, de la fin des années 1970 à nos jours, et représentative des concentrations en surface des $\mathrm{PM}_{10}$ au Sahel, en hiver, a été réalisée en utilisant l'AI-OMI validé sur la période 2006-2009 comme référence. En outre, l'AI-OMI de 2006 à 2009 est pondéré par un indice basé sur le rapport $\mathrm{PM}_{10} / \mathrm{AI}$ dont le cycle saisonnier est marqué pour les deux sites sahéliens considérés (Cinzana au Mali et Banizoumbou au Niger), avec des valeurs proches de zéro en saison des pluies et des valeurs variant de 500 à 2000 en plein cœur de la saison sèche. Ainsi, c'est la tendance saisonnière moyenne du rapport $\mathrm{PM}_{10} / \mathrm{AI}$ au pas de temps hebdomadaire qui est utilisée pour pondérer les AI-OMI de 2006 à 2009. Ils sont ensuite utilisés pour recaler les données TOMS dotées d'une résolution spatiale moins fine. La série continue TOMS/OMI obtenue n'est ni un $\mathrm{AI}$ ni un « proxy » des $\mathrm{PM}_{10}$ en surface mais bien un indice « aérosol» spatialisé qui, compte tenu de l'utilisation des données sol acquises dans AMMA, est plus représentatif de la réalité des aérosols circulant dans les basses couches atmosphériques en plein cœur de l'hiver. Cette méthodologie, décrite dans Agier et al. (2012), constitue un premier pas vers les études d'impacts sur la santé sur le long terme (voir la dernière section).

\section{Exploitation des observations micro-onde sur le continent africain pour la météorologie}

L'étude d'un environnement complexe comme le système de mousson en Afrique de 1'Ouest nécessite une connaissance de l'ensemble des conditions de surface et de l'atmosphère. Ces dernières sont souvent déduites des analyses ou des prévisions à courte échéance ou bien des réanalyses sur plusieurs décennies provenant des modèles de prévisions numériques du temps (PNT). Jusqu'à récemment, peu d'observations en Afrique de l'Ouest, où le réseau de mesures in situ est très peu dense, ont été utilisées dans les modèles de prévision, soulignant l'importance du renforcement du réseau de radiosondage, ainsi que de nouveaux développements pour améliorer l'utilisation des observations spatiales. En particulier, une nouvelle approche a été mise au point pour exploiter la radiométrie micro-onde au-dessus de l'Afrique en PNT par le biais d'une modélisation plus adaptée de l'émissivité des surfaces continentales (Karbou et al., 2010a et b ; Gérard et al., 2010). Karbou et al. (dans ce numéro, p. 49-54) revient plus en détail sur les progrès considérables réalisés en prévision numérique du temps dans le cadre de AMMA.

Toutefois, il peut se passer des mois, voire des années, entre la mise au point d'un développement scientifique et son portage vers l'opérationnel dans les modèles de PNT, et les données satellitaires peuvent permettre d'évaluer les réanalyses. Les mesures des instruments AMSU-A et -B (Advanced Microwave Sounding Unit-A et -B) nous renseignent sur la structure verticale de la température (de la surface jusqu'à $45 \mathrm{~km}$ d'altitude environ) et de l'humidité (de la surface à une vingtaine de kilomètres d'altitude). Les mesures AMSU sont acquises avec un angle d'observation variant de $-58^{\circ}$ à $+58^{\circ}$ par rapport au nadir, induisant des variations dues à l'épaisseur atmosphérique et la surface. Pour utiliser ces données, une méthode de correction empirique de l'effet de l'angle d'observation a été développée. La figure 3 a montre un exemple de cartes moyennes de températures de brillance (Tbs) du canal 5 d'AMSU-A. Ce dernier renseigne sur la température dans la basse troposphère, jusqu'à environ 4-5 km d'altitude. On peut clairement distinguer la trace des orbites, par l'effet de l'angle d'observation (valeurs de Tbs plus fortes à la verticale que sur les bords de l'orbite). La figure $3 \mathrm{~b}$ illustre l'effet de la correction au nadir de ces mêmes données : les structures principales de l'atmosphère et de la surface sont maintenant identifiables (en particulier la région saharienne, où se développe en été une dépression 

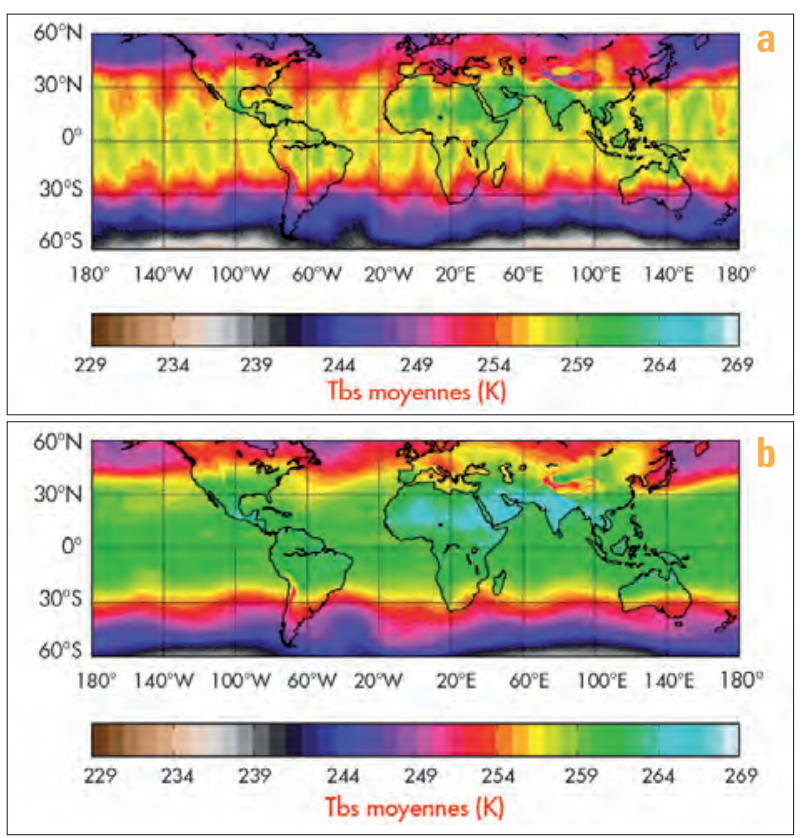

thermique importante pour la mousson). L'application de cette correction permet ainsi d'interpréter directement les mesures pour des études de variabilité du climat.

\section{Surface continentale et végétation}

L'utilisation conjointe des observations de terrain et des données satellitaires a permis de d'évaluer et de valider un bon nombre des « variables essentielles pour le climat », définies par les institutions internationales comme l'OMM (Organisation météorologique mondiale) ou la FAO (Food and Agriculture Organization); on se référera à Kergoat et al. (2011) pour un résumé exhaustif. Les termes du bilan radiatif tout d'abord, comme l'albédo de surface (Samain et al., 2008), les rayonnements solaire et infrarouge à la surface sont estimés à l'aide des instruments visible et infrarouge des satellites météorologiques défilants ou géostationnaires. Ces variables ont été examinées en détail sur un domaine de latitude et de l'océan aux abords du Sahara et confrontées aux mesures au sol. La présence spectaculaire des poussières désertiques a été identifiée comme un facteur critique qui nécessite une meilleure prise en compte dans l'estimation des flux radiatifs à la surface, aussi bien pour les produits satellitaires que pour les modèles (PNT ou climat).

Les paramètres décrivant la végétation, comme l'indice foliaire (la surface de feuille par mètre carré de sol), bénéficient de la capacité des capteurs spatiaux à rendre compte avec précision de la variabilité spatiale et temporelle.
Figure 3 - Cartes moyennes des températures de brillance pour le mois de juin 2011 issues du canal 5 de l'instrument AMSU-A, (a) sans et (b) avec correction de l'effet de l'angle d'observation.

Chaque année, ces capteurs identifient les zones frappées par une sécheresse ou au contraire profitant d'une bonne saison des pluies, ce qui est d'un intérêt immédiat pour le suivi des ressources agricoles, pastorales et pour les services d'alerte précoce. L'humidité des sols, paramètre important également, par exemple pour le déclenchement de la convection (Guichard et al., ce numéro, p. 25-32) peut maintenant être correctement déduite des mesures spatiales dans les zones à faible couvert végétal (voir section « Calipso : nuages et aérosols » ci-dessus ; Baup et al., 2007 ; Zribi et al. ; 2007). La sensibilité du signal radar a été démontrée, par exemple, à l'aide de données recueillies dans le Ferlo (Sénégal) par le Centre de suivi écologique (Diouf et al., 1998 ; Faye et al., 2011 ; Riazanoff et al., 2009). Le signal radar des capteurs SCAT/ASCAT de ERS et METOP crô̂t avec l'augmentation de la teneur en eau du sol d'une part, et de l'évolution de la densité et du contenu en eau de la couche herbacée d'autre part (figure 4).

Une autre « variable essentielle pour le climat », l'évapotranspiration, qui est un terme très important du bilan hydrique, peut également être déduite des données infrarouge et optiques des satellites défilants ou géostationnaires (par exemple Ridler et al., 2012 ; Tanguy et al., 2012). À plus grande échelle, le satellite GRACE permet d'accéder aux variations temporelles du stock d'eau total, comprenant la zone non saturée, les étendues d'eau et les nappes (Grippa et al., 2011), en détectant les changements du champ de gravité terrestre causés par la variation de ces masses d'eau.

Enfin, à 1'échelle de l'Afrique de l'Ouest, Kaptué et al. (2010) ont développé une nouvelle cartographie des paramètres de surface, ECOCLIMAP2. Ce jeu de données, spécifique à l'Afrique, a été utilisé au cours de l'intercomparaison des schémas de surface des modèles de PNT et de climat (Boone et al., 2009).

\section{Exploitation scientifique des observations spatiales}

Les données spatiales, après traitement approprié et validation, permettent d'analyser des processus et mécanismes du milieu ou d'étudier la variabilité du système. Elles sont utilisées seules ou, le plus souvent, en complément d'observations in situ et de simulations numériques.

\section{Étude des émissions et du transport des poussières à l'échelle régionale}

L'utilisation des nouvelles mesures spatiales de télédétection active (i.e. le lidar CALIOP) a permis de mettre en évidence un ensemble de mécanismes dynamiques nouveaux contrôlant la structure de la couche limite atmosphérique saharienne ainsi que sa composition en aérosols, au cours de la saison chaude : ressauts hydrauliques à proximité des reliefs et circulations secondaires associées à un chauffage différentiel en surface et aux anomalies d'albédo de surface (Cuesta et al., 2009). Elle a

Figure 4 - Coefficient de rétrodiffusion mesuré par les capteurs de ERS (1992-2007), en bleu, et ASCAT de METOP (2007-2008), en rouge, à $45^{\circ}$ d'incidence au Ferlo (Sénégal). Les cumuls de pluie quotidiens mesurés par les pluviomètres sont superposés (en vert). D'après Faye et al. (2011).

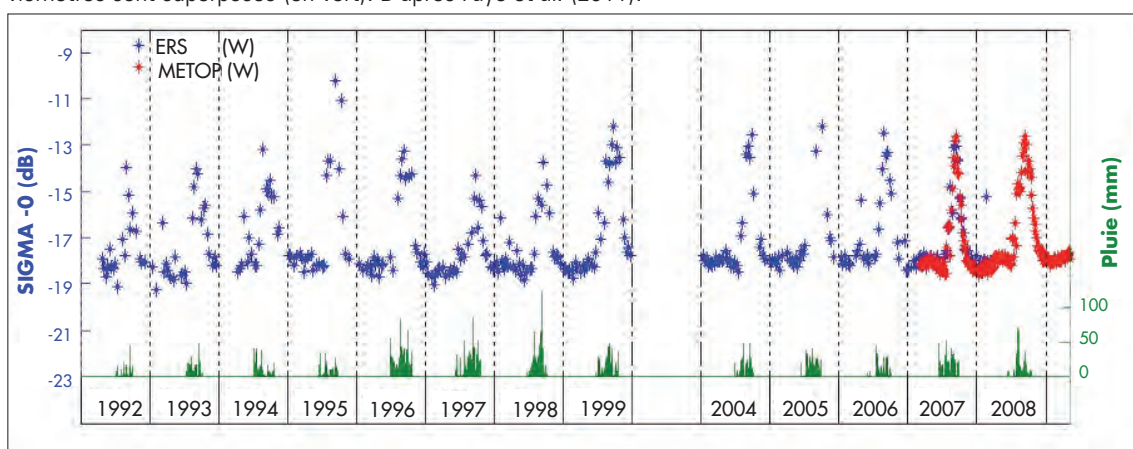


également permis d'identifier les soulèvements de poussières désertiques liés au front d'harmattan pendant la saison sèche (Haywood et al., 2008) et la validation des simulations numériques de soulèvement et de transport de poussières associés à de nombreux processus étudiés pendant AMMA : front de mousson (Bou Karam et al., 2009a), cyclones se formant au niveau de la discontinuité intertropicale (Bou Karam et al., 2009b) ou en aval des montagnes de l'Atlas (Bou Karam et al., 2010).

\section{Rétroactions surface- atmosphère : influence de l'humidité des sols sur les précipitations}

L'évapotranspiration de surface modifie les conditions de température et d'humidité des basses couches de l'atmosphère et agit en conséquence sur le développement de la convection. L'hypothèse selon laquelle les conditions de surface et, en particulier, l'humidité du sol jouent un rôle sur la circulation atmosphérique et le régime des précipitations a été proposée bien avant le programme AMMA. Plusieurs études ont montré par le biais de la modélisation (Zheng et al., 1999 ; Koster et al., 2004) et des observations (Taylor et Lebel, 1998 ; Philippon et al., 2002) l'existence d'un lien entre précipitations et humidité du sol, le Sahel du globe où cette rétroaction est la plus forte. La raison est relativement simple : pour que les interactions surfaceatmosphère soient un phénomène prépondérant, il faut une évaporation apparaissant comme l'une des régions

suffisamment abondante pour agir sur les précipitations mais suffisamment faible pour être contrôlée par l'humidité du sol, ce qui exclut les climats humides et les climats trop secs au profit des régions de transition entre ces deux types de climats.

Pourtant, il reste aujourd'hui une réelle incertitude sur la force et le signe de cette rétroaction, incertitude liée en grande partie à la qualité des informations d'humidité du sol utilisées. Ainsi, il ressort de certaines études qu'une augmentation de l'humidité du sol tend à augmenter les précipitations tandis que d'autres concluent à un effet négatif de l'humidité du sol. Dans ce cadre, l'utilisation de la télédétection spatiale sur l'Afrique de l'Ouest a permis d'obtenir des résultats significatifs. Parmi d'autres, Taylor et al. (2011b) ont utilisé conjointement un produit de suivi de systèmes convectifs basé sur Météosat, un produit d'humidité du sol (AMSR$\mathrm{E} / A Q U A)$ et de températures du sol $(M S G)$ afin d'examiner le rôle de l'humidité du sol dans le déclenchement de la convection. Ils ont montré que les systèmes convectifs se déclenchent préférentiellement sur des zones sèches à proximité de zones humides et dans une configuration de vent particulière (Guichard et al., ce numéro, p. 25-32).

Outre le déclenchement, il semble que la trajectoire et le comportement des systèmes convectifs au cours de leur vie soient influencés par les structures d'humidité du sol comme l'illustre la figure 5. La trajectoire du système qui se déclenche sur l'ouest du Mali à $2 \mathrm{~h}$ le 26 juillet 2006 semble corrélée à la

Figure 5 - Exemple d'une rétroaction surface-atmosphère sur le système convectif du 26 juillet 2006. Les contours successifs du système convectif (en rouge) sont issus du produit ISIS de Météo-France et se basent sur les mesures de Météosat. La carte d'humidité du sol (zones grisées) est basée sur un algorithme couplant un produit satellitaire de précipitation (CMORPH) et des mesures micro-onde (AMSR-E). Le système semble évoluer en trois phases, une phase d'expansion entre $2 \mathrm{~h}$ et $9 \mathrm{~h}$, une phase de décroissance entre $11 \mathrm{~h}$ et $14 \mathrm{~h}$, puis à nouveau une expansion lorsque le système atteint la côte Atlantique. La phase de décroissance semble liée à la présence de deux zones humides au nord (Sénégal) et au sud (Guinée).

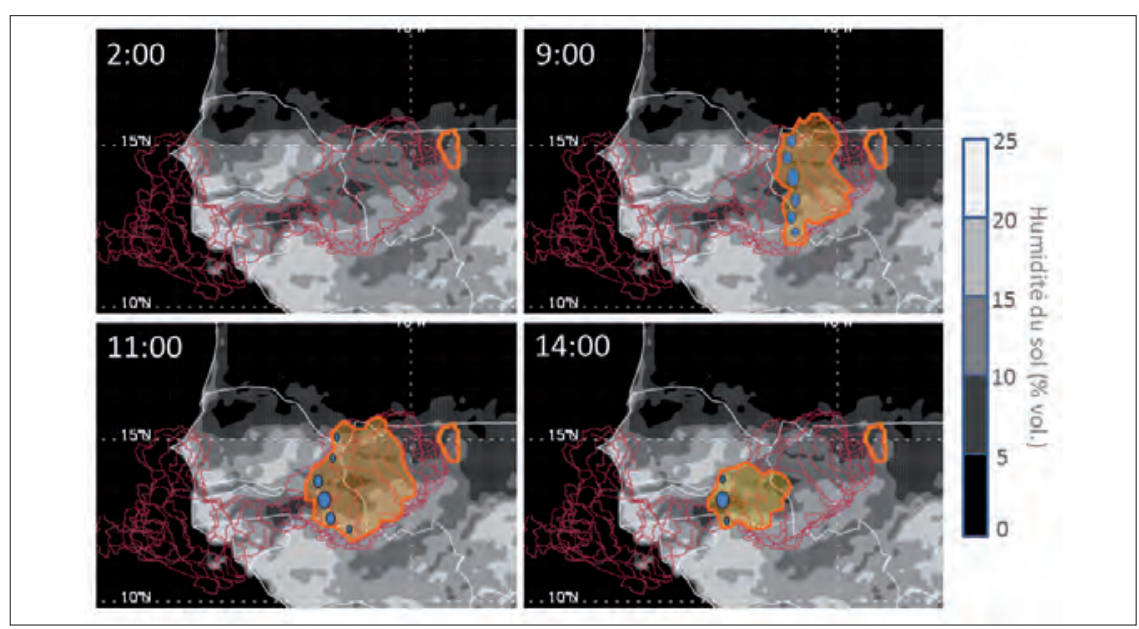

présence de deux zones humides au nord (Sénégal) et au sud (Guinée). L'hypothèse formulée est que les cellules convectives, qui composent le système de mésoéchelle (en bleu sur la figure 5), sont influencées par leur environnement immédiat ; les cellules évoluant sur les zones sèches bénéficient d'ascendances thermiques alors que les cellules évoluant sur les zones humides rencontrent des sols plus froids donc moins générateurs de convection. Une étude systématique, basée sur plus de 5000 systèmes convectifs, a été initiée (article en préparation par Pellarin et al.).

\section{Climatologie et variabilitité intra-saisonnière de la mousson}

La plupart des études sur le milieu naturel sont fondées sur l'utilisation de « produits » résultant de l'élaboration de relations entre variables du milieu et mesures spatiales (par exemple précipitations, humidité du sol...). Cependant, ces méthodes sont imparfaites, en raison des incertitudes sur les outils statistiques et les bases de données utilisés, et ne sont pas valables en toutes conditions. Une limitation importante à l'utilisation des sondeurs micro-onde de température et d'humidité atmosphériques est la présence de nuages et de pluie. Pour s'affranchir de ces limitations, Eymard et al. (2010) ont exploité directement les mesures de température de brillance des sondeurs AMSU-A et -B. Pour cela, la méthode de correction angulaire des mesures décrite précédemment a été appliquée, puis les mesures ont été interpolées pour créer une base de données AMSU sur 8 ans (résolution $1^{\circ}$ et 1 jour). La comparaison avec les réanalyses ERA-Interim (Simmons et al., 2007) a été effectuée de façon détaillée, tant à l'échelle intra-saisonnière que de façon climatologique. L'analyse de cette climatologie a révélé que les mesures AMSU, une fois corrigées de l'effet de l'angle d'observation, permettent d'observer certaines caractéristiques de la mousson ouest africaine : déplacement de la zone de convergence intertropicale, suivi du développement de la dépression thermique saharienne dans la basse troposphère. Elle ont permis de mettre en évidence l'existence de zones très sèches, notamment dans la région est du continent, structures très mal connues et qui sont très mal représentées dans les modèles de PNT. De plus, les analyses ne représentent pas correctement les modes de variabilité intra-saisonnière (périodicité dominante de 15 jours environ), qui sont mises en évidence dans les données. 


\section{Interactions océan- atmosphère}

La télédétection spatiale apporte des informations indispensables pour fournir aux modèles numériques océaniques leurs conditions limites de surface (le " forçage ») et des corrections en surface (l'assimilation), particulièrement dans les régions équatoriales où les données sont rares. Les observations dans les régions spectrales visible, infrarouge et micro-onde permettent de disposer de flux radiatifs (principale composante du bilan net de surface sur les océans tropicaux à égalité avec le flux turbulent de chaleur latente), des températures de surface de la mer (signal saisonnier le plus fort sur le bassin Atlantique, maximum des tropiques au Sénégal), ou encore de la réflectance de l'océan, signature d'absorption chlorophyllienne qui influence la pénétration du rayonnement solaire dans les couches de surface. Grâce aux mesures par diffusiométrie, vitesses et directions du vent sont obtenues, tandis que les variations de hauteur de la mer sont détectées par altimétrie, avec une précision de l'ordre du centimètre. Les champs de précipitation, même s'ils n'ont pas encore la résolution spatiale suffisante pour des études de mésoéchelle, sont un élément particulièrement sensible pour étudier l'océan Atlantique tropical.

Les mesures in situ de la campagne AMMA/EGEE-3 (de fin mai à début juillet 2006 dans le golfe de Guinée ; Lebel et al., 2009), ont montré que de réels progrès restaient encore à accomplir dans les restitutions des flux radiatifs déduits des mesures de $M S G$ pour prendre en compte l'effet des aérosols (Le Borgne et al., 2007) et que les précipitations sur l'océan devaient faire l'objet d'investigations supplémentaires. Athié et Marin (2008) montrent en revanche que les champs de tension du vent de surface déduits des mesures spatiales (diffusiomètre du satellite QuickSCAT) permettent de simuler correctement des structures océaniques au voisinage de l'équateur et que les ondes tropicales d'instabilité ont les bonnes vitesses dans les modèles numériques.

Les données spatiales (vent QuickSCAT, température de surface de l'océan et vapeur d'eau TMI, précipitations TRMM, couverture nuageuse $M S G$, hauteur de la mer Jason) ont joué un rôle essentiel pour un grand nombre d'études de processus et de variabilité du couplage océan-atmosphère, soit directement, soit à travers la validation des réanalyses des modèles globaux du
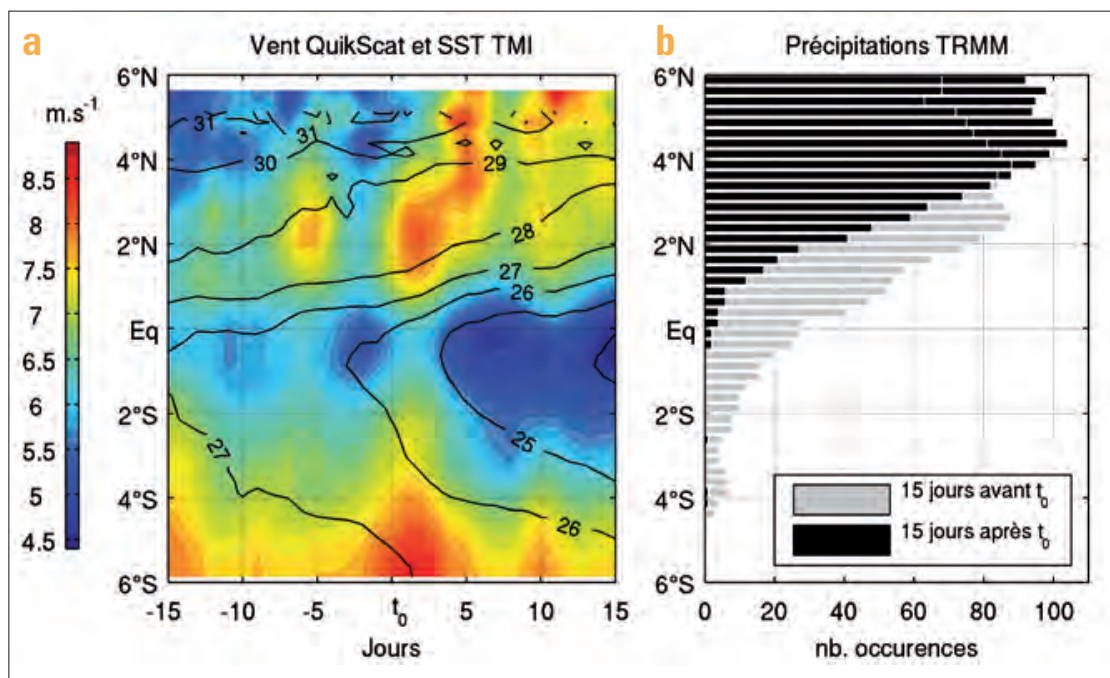

Figure 6 - Changement de régime de la mousson de printemps et impact sur les précipitations. Les coups de vent de sud se succèdent durant le printemps boréal, contribuant aux refroidissements successifs de la langue d'eau froide à l'équateur. Chaque année, on observe un changement de régime, caractérisé par un vent toujours plus fort au nord du golfe de Guinée qu'à l'équateur. a) (Échelle de couleur), champ de vent (QuickSCAT) de 15 jours avant à 15 jours après la date de référence correspondant à ce changement de régime (moyenne sur 10 ans) : le vent augmente de $2 \mathrm{~m} \mathrm{~s}$ au nord du golfe et ralentit sur la langue d'eau froide. En contours superposés (lignes noires), on montre l'évolution correspondante de la température de surface, caractérisée par un net refroidissement à l'équateur. b) Impact de ce changement de régime sur l'occurrence des précipitations (base de données TRMM, Huffman et al, 2007) : le maximum d'occurrence de précipitation est décalé de 2 degrés vers le nord à partir de cette date.

Centre européen pour les prévisions météorologiques à moyen terme (CEPMMT) et du National Center for Atmospheric Research (NCAR) :

- la réponse de la couche mélangée océanique équatoriale aux forçages atmosphériques a été mise en évidence par Wade et al. (2011a et b), montrant l'importance du mélange turbulent sur le refroidissement dans la langue d'eau froide ; Polo et al. (2008), Wade et al. (2012) ont établi le rôle de la propagation d'ondes côtières depuis l'équateur sur la modulation de la température de surface de l'océan (jusqu'à $\pm 0,5^{\circ} \mathrm{C} / \mathrm{cm}$ ) le long des côtes ouestafricaines ;

- l'océan équatorial dans le golfe de Guinée rétroagit sur le flux de mousson par la formation d'une langue d'eau froide à l'équateur qui modifie le régime de vent de basses couches alimentant la zone de mousson au printemps boréal (Coëtlogon et al., 2010 ; Caniaux et al., 2011). En utilisant des indices issus de

bases de données satellitaires (précipitations, températures de surface), Nguyen et al. (2011) confirment que la phase côtière de la mousson est très corrélée à la variabilité de la langue d'eau froide. À partir de vents et de températures de surface de l'océan restitués par satellite, LeducLeballeur et al. $(2011,2012)$ analysent le fonctionnement des épisodes de coup de vent et la rétroaction de l'océan : le front équatorial qui marque la limite nord de la langue d'eau froide joue un rôle clef dans les processus de couplage qui modulent les précipitations côtières et influencent la période du saut de mousson (figure 6).

\section{Suivi des eaux de surface continentales (mares)}

La profondeur des archives satellitaires, qui atteint maintenant 45 ans pour les premiers satellites, 40 ans pour Landsat, 30 ans pour Météosat et la série des NOAA, permet d'aborder la variabilité

Figure 7 - Évolution de la mare d'Agoufou au Mali, délimitée par le contour rouge à partir de données déclassifiées Corona de septembre 1966 (à gauche, image en noir et blanc), de photographies aériennes d'octobre 1996, (au centre, noir et blanc, la mare paraît claire à cause de la réflexion du soleil à la surface de l'eau) et d'images Landsat d'octobre 1999, (à droite, image multispectrale). La mare, qui n'était encore en 1966 qu'un bas-fond occupé par un bosquet d'arbre, s'est progressivement étalée, les arbres sont morts asphyxiés et, en 1990, la mare est devenue une mare permanente qui atteint maintenant 250 ha. Ce phénomène est une conséquence inattendue de la sécheresse des quatre dernières décennies.

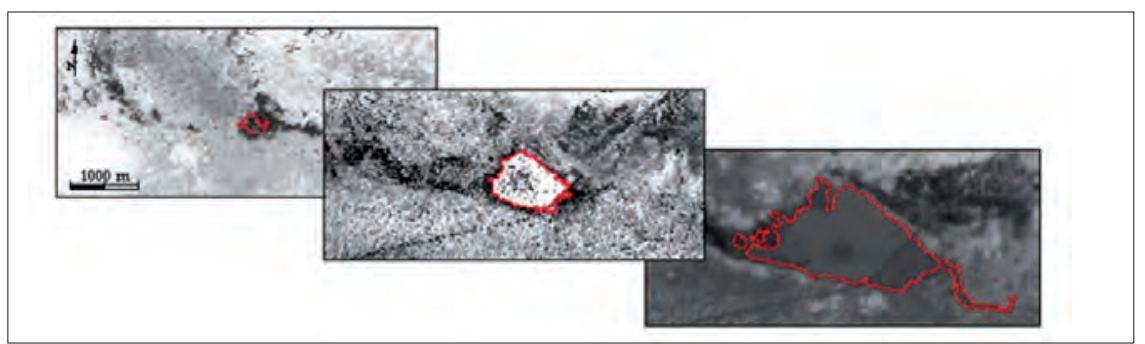


multi-décennale de l'environnement. On a pu montrer par exemple que la sécheresse intense des années 1970 à nos jours, qui a atteint son maximum en 1973 et 1984, s'est accompagnée d'une augmentation paradoxale des niveaux d'eau dans les mares et des écoulements en région sahélienne, malgré des pluies inférieures à celles des années 1950 (Gardelle et al., 2010), comme le montre la figure 7 . Le phénomène inverse, c'est-à-dire une réduction du ruissellement, est observé en zone soudanienne, ce qui montre la complexité de la réponse des surfaces continentales aux variations du climat.

\section{Développements d'applications sociétales utilisant les données spatialles}

Les sections précédentes présentent les travaux de recherche utilisant la télédétection spatiale pour améliorer la compréhension du fonctionnement du milieu naturel. Mais un autre atout des mesures de télédétection spatiale est la possibilité de surveiller le milieu et d'obtenir des indicateurs pertinents dans d'autres domaines, comme les épidémies de maladies infectieuses, les ressources en eau et l'agriculture, ou l'état des écosystèmes.

\section{Relations}

\section{aérosols/méningites}

L'intensité ainsi que le calendrier des méningites semblent très liés aux variables climatiques vent et humidité. En outre, il semble que la variabilité du nombre de cas entre le démarrage et le pic du cycle saisonnier des méningites soit très liée à la variabilité des événements d'aérosols désertiques (Martiny et al., dans ce numéro, p. 73-79).
Ainsi, les auteurs observent systématiquement un pic d'aérosols avant chaque pic du nombre de cas de méningites sur la période allant du démarrage au maximum du cycle saisonnier avec un décalage moyen de 2 semaines. Cette relation aérosols/méningite semble s'interrompre avec la phase d'augmentation de l'humidité liée à l'arrivée de la mousson. En effet, le cycle saisonnier des aérosols s'arrête bien plus tardivement que celui des méningites mais les événements de poussières survenant après la semaine 13 ne semblent plus jouer de rôle sur le nombre de cas de méningite. L'AI-OMI à une résolution spatiale de $0,25^{\circ}$ étant adapté aux études d'impacts sur les épidémies de méningite et spécifiquement au pas de temps hebdomadaire (Deroubaix et al., 2012) a été utilisé pour conforter ces hypothèses.

En effet, le tableau 1 montre que les corrélations entre AI-OMI et nombre de cas de méningite sont meilleures en considérant la période du démarrage au maximum du cycle saisonnier (colonne $\mathrm{C} 1$ ) et pour un décalage de 2 semaines. Ces résultats, qui confortent des recherches précédemment réalisées avec d'autres jeux de données, témoignent en outre d'avancées significatives dans l'utilisation des données de missions spatiales pour les études de l'impact de l'environnement sur la santé.

\section{Télédétection spatiale et agriculiture}

Les données de télédétection sont de plus en plus utilisées pour suivre l'état des cultures à mesure que la saison des pluies progresse. Des systèmes d'alerte précoce permettent notamment d'identifier à l'avance les zones susceptibles d'avoir un déficit en rendement agricole et donc d'anticiper des crises alimentaires. Le programme AMMA a contribué à l'amélioration de ces systèmes d'alerte précoce à travers un travail sur le modèle agrométéorologique Zones à risque (Bacci et al., 2010 ; Genesio et al., 2006) qui utilise les champs d'estimation de précipitations à 10 jours dérivés de $M S G$ pour faire un diagnostic en temps réel de l'état des cultures. Outre son apport pour suivre l'état des cultures, la télédétection est un outil essentiel pour l'estimation des surfaces cultivées, particulièrement problématique en raison d'un domaine cultivé fragmenté, d'une grande hétérogénéité spatiale due aux conditions environnementales et aux pratiques culturales, et de la synchronisation des phénologies des agrosystèmes et des écosystèmes liée au régime des précipitations. Vintrou et al. (2011 et 2012) ont proposé des développements méthodologiques originaux pour la caractérisation des systèmes agricoles d'Afrique de l'Ouest par télédétection. Les méthodes ont été développées à partir de séries temporelles MODIS (250 m à $500 \mathrm{~m}$ de résolution spatiale) acquises sur le Mali. Dans un premier temps, la cartographie du domaine cultivé a été réalisée à partir d'indices spectraux, spatiaux, texturaux et temporels dérivés des images MODIS. Dans un second temps, le domaine cultivé a pu être caractérisé en distinguant trois grands types de systèmes agricoles à l'échelle villageoise : céréales dominantes (mil, sorgho), cultures intensives dominantes (maïs, coton) et mélange de sorgho et de coton (figure 8). Cette deuxième étape a mobilisé les indicateurs de télédétection précédemment cités, ainsi qu'une base de données d'enquêtes de terrain de l'Institut d'économie rurale de Bamako (Mali) sur 100 villages.

Ce travail a permis de dégager de nouvelles pistes de couplage entre télédétection, données de terrain et modélisation agrométéorologique en apportant une information continue dans le temps et dans l'espace sur la caractérisation du domaine cultivé en Afrique de l'Ouest.

Tableau 1 - Coefficients de corrélation entre l'AI-OMl et le nombre de cas de méningite en considérant différents temps de décalage (de 0 à 4 semaines) et deux périodes distinctes (C1 : du démarrage au maximum du cycle saisonnier ; C2 : du démarrage à la fin du cycle saisonnier de méningite).

\begin{tabular}{|c|c|c|c|c|c|c|}
\hline Pays & \multicolumn{2}{|c|}{ Burkina Faso } & \multicolumn{2}{|c|}{ Niger } & \multicolumn{2}{|c|}{ Mali } \\
\hline Périodes & Cl & $\mathrm{c} 2$ & C1 & $\mathrm{C} 2$ & C1 & $\mathrm{C} 2$ \\
\hline Aucun & 0,18 & 0,20 & 0,73 & $-0,39$ & 0,79 & -060 \\
\hline 1 semaine & 0,12 & 0,32 & 0,63 & $-0,51$ & 0,85 & $-0,62$ \\
\hline 2 semaines & 0,81 & 0,77 & 0,90 & $-0,46$ & 0,91 & $-0,55$ \\
\hline 3 semaines & 0,64 & 0,63 & 0,72 & $-0,55$ & 0,80 & $-0,62$ \\
\hline 4 semaines & 0,47 & 0,47 & 0,80 & $-0,56$ & 0,55 & $-0,61$ \\
\hline
\end{tabular}




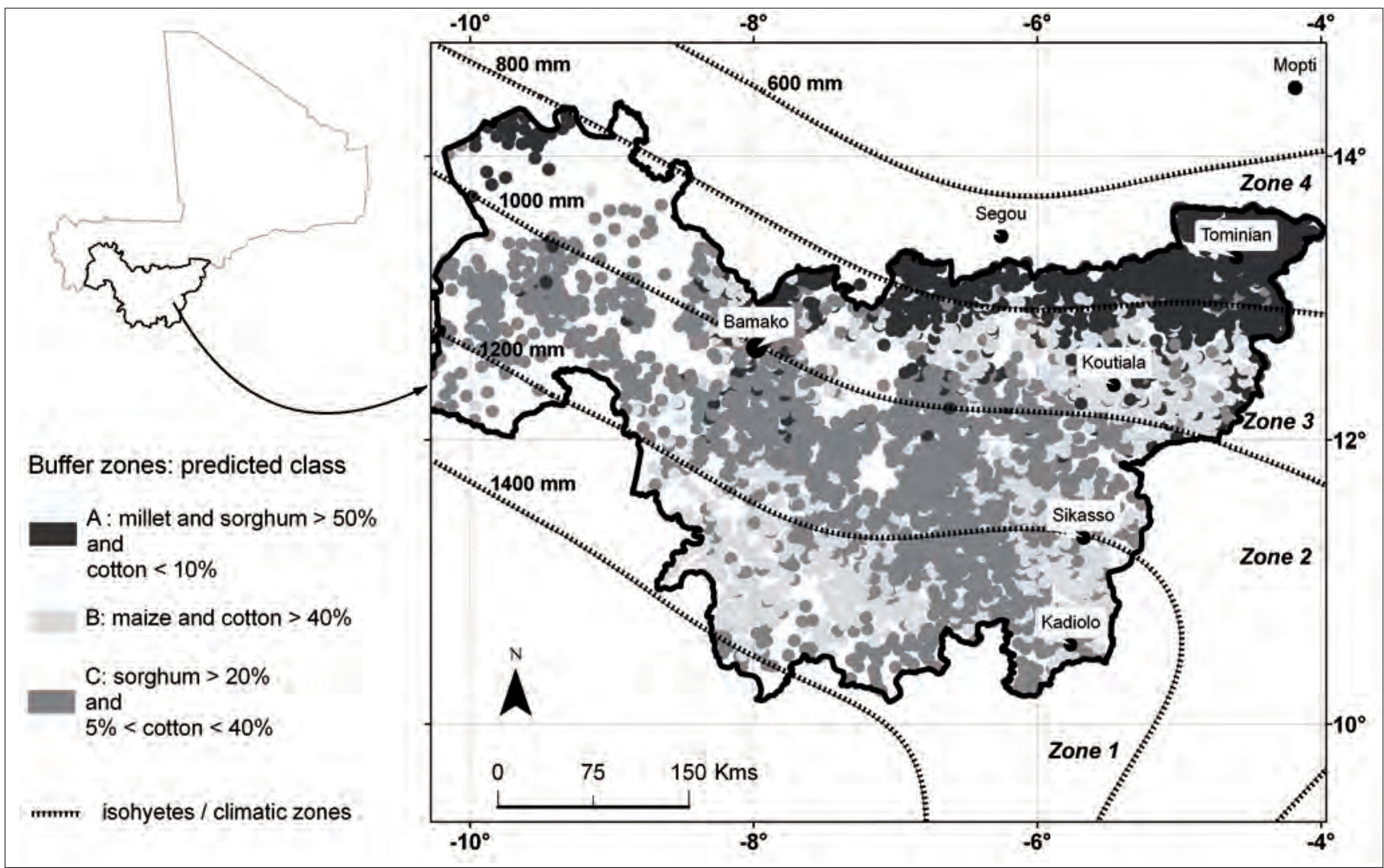

Figure 8 - Systèmes agricoles villageois dans le sud du Mali (A : agriculture de subsistance, B : agriculture intensive, C : système mixte).

\section{Ecosystèmes et mousson}

Au Sahel, la saison de mousson est en général le seul apport pluvial pour la végétation. Le cycle de végétation est de ce fait très marqué. Bobée et al. (2010) ont montré la possibilité d'utiliser le Leaf Area Index (LAI) comme indicateur des précipitations «efficaces», en corrélant les données de pluie locale avec le LAI MODIS sur la période 2000-2008 à échelle fine sur la Grande Côte (Sénégal).

Le type de sol est déterminant dans l'évolution du LAI. En effet, ces mêmes auteurs ont trouvé que les sols ferrugineux tropicaux présentent les valeurs les plus élevées de LAI, alors que les plus faibles valeurs du LAI sont associées aux faibles densités de la végétation enregistrées sur les sols minéraux bruts. Les deux indicateurs que sont la fréquence de distribution des événements pluvieux (accessible depuis l'observation satellitaire) au cours de la période de mousson et la combinaison des seuils de précipitations cumulées, corrélés avec les dates d'apparition ou de stress hydrique de la végétation sont pertinents pour prévoir l'évolution de la végétation (Cissé et al., 2012). Ils pourraient être appliqués à d'autres régions et en utilisant des scénarios climatiques pour faire des prévisions de changement dans la croissance de la végétation ainsi que de la productivité.

\section{Conclusion}

La télédétection spatiale a été très tôt identifiée comme un complément indispensable aux mesures locales dans la stratégie AMMA. Elle a permis des avancées tant pour la maîtrise des données spatiales elles-mêmes (validation des mesures des capteurs et des « produits » atmosphériques ou de surface déduits, nouvelles méthodes d'utilisation), que sur les mécanismes du système de mousson, dans l'atmosphère et sur les surfaces continentale et océanique.

L'enseignement général tiré de AMMA est que les capteurs spatiaux les plus récents fournissent une information extrêmement solide pour un grand nombre de variables essentielles pour le climat, base sur laquelle les modèles de climat peuvent être évalués et étalonnés.

Un second enseignement est qu'il est important de maintenir des réseaux d'observation en Afrique de l'Ouest pour progresser sur les questions spécifiques à cette région du monde comme l'influence des poussières désertiques ou l'évolution de l'usage des sols par exemple. En effet, quels que soient les progrès accomplis dans l'exploitation des missions spatiales, il reste impossible de décrire l'ensemble des variables du milieu.
Enfin, plusieurs études récentes montrent que la télédétection, au-delà des études du milieu naturel (système climatique, hydrologie continentale, végétation, océan), peut contribuer aux travaux sur les impacts de la variabilité climatique et intervenir dans la construction de systèmes d'alerte pour l'agriculture, les ressources en eau et la santé. Les prochaines années devraient voir émerger de plus en plus d'applications sociétales associant données spatiales (au-delà de la cartographie déjà largement utilisée), données in situ et modélisation numérique.

\section{Remerciements}

Cet article a été écrit avec la contribution de : Agali Alhassane, Mauricio Bacci, Agnès Begué, Dominique Bouniol, Gaëlle de Coëtlogon, Juan Cuesta, Julien Delanoë, Adrien Deroubaix, Arona Diedhou, Thomas Fiolleau, Lorenzo Genesio, Marielle Gosset, Manuela Grippa, Claire Gruhier, Roselyne Lacaze, Alban Lazar, Marion Leduc-Leballeur, Éric Mougin, Catherine Ottle, Jan Polcher, Patricia de Rosnay, Benjamin Sultan, Sibiry Traoré, Nicolas Viltard, Malick Wade, Mehrez Zribi. Les auteurs remercient l'équipe «BDD AMMA » pour son aide pour la fourniture des données spatiales et la mise à disposition des résultats. 